\title{
Lidar: a new self-driving vehicle for introducing optics to broader engineering and non-engineering audiences
}

\section{Corneliu Rablau}

Corneliu Rablau, "Lidar: a new self-driving vehicle for introducing optics to broader engineering and non-engineering audiences," Proc. SPIE 11143, Fifteenth Conference on Education and Training in Optics and Photonics: ETOP 2019, 111430C (2 July 2019); doi: 10.1117/12.2523863 


\title{
LIDAR - A new (self-driving) vehicle for introducing optics to broader engineering and non-engineering audiences
}

\author{
Corneliu Rablau ${ }^{\mathrm{a}}$ \\ ${ }^{a}$ Kettering University, Dept. of Physics, 1700 University Ave., Flint, MI USA 48504
}

\begin{abstract}
Since Stanley, the self-driven Stanford car equipped with five SICK LIDAR sensors won the 2005 DARPA Challenge, the race to developing and deploying fully autonomous, self-driving vehicles has come to a full swing. By now, it has engulfed all major automotive companies and suppliers, major trucking and taxi companies, not to mention companies like Google (Waymo), Apple and Tesla. With the notable exception of the Tesla self-driving cars, a LIDAR (Light, Detection and Ranging) unit is a key component of the suit of sensors that allow autonomous vehicles to see and navigate the world. The market space for lidar units is by now downright crowded, with a number of companies and their respective technologies jockeying for long-run leading positions in the field. Major lidar technologies for autonomous driving include mechanical scanning (spinning) lidar, MEMS micro-mirror lidar, optical-phased array lidar, flash lidar, frequencymodulated continuous-wave (FMCW) lidar and others. A major technical specification of any lidar is the operating wavelength. Many existing systems use $905 \mathrm{~nm}$ diode lasers, a wavelength compatible with CMOS-technology detectors. But other wavelengths (like $850 \mathrm{~nm}, 940 \mathrm{~nm}$ and $1550 \mathrm{~nm}$ ) are also investigated and, in the long run, the telecom nearinfrared range $(1550 \mathrm{~nm})$ is expected to experience significant growth because it offers a larger detecting distance range (200-300 meters) within eye safety laser power limits while also offering potential better performance in bad weather conditions. This paper discusses the above-mentioned technical (optics and photonics) aspects of the most common lidar technologies, with the educational focus of identifying opportunities for employing such discussions in introducing optics to broader engineering audiences, drawing in part on experiences and examples from Kettering University.
\end{abstract}

Keywords: LIDAR, self-driving, autonomous vehicles, AutoDrive ${ }^{\mathrm{TM}}$ Challenge

\section{INTRODUCTION}

\subsection{The race to a fully autonomous (Level 5) self-driving vehicle is on}

Founded in 1919 as an automotive-trades company that soon became General Motors Institute of Technology, Kettering University is a private, primarily undergraduate university located in Flint, MI, not far from the Detroit Metropolitan Area - the heart of the US automotive industry. The university holds a strong presence in both traditional and self-driving automotive technologies education, training and research through its Mechanical Engineering, Electrical and Computer Engineering, and Computer Science programs. At the same time, it maintains a healthy presence in the optics education area through its Applied Optics minor offered by the Applied Physics and Engineering Physics programs. Kettering's Flint campus is home of the Kettering University GM Mobility Research Center [1], a vehicle and mobility systems development proving ground and outdoor research facility. This one-of-its-kind facility on a college campus in the country puts Kettering and Flint on the global map of the autonomous vehicle race. In 2017, Kettering University became one of only eight universities in North America selected to participate in the Society of Automotive Engineers' (SAE) AutoDrive ${ }^{\mathrm{TM}}$ Challenge [2] - an autonomous vehicle inter-collegiate competition. This inter-collegiate competition represents a microcosm of the intense competition currently unfolding at an accelerated pace and on a global scale for development of technologies and systems for autonomous vehicles in what already shapes up to be a huge area of economic growth and societal impact in the next 30 years and beyond. With lidar a key component of those technologies, a unique set of circumstances have been created for delivering optics and photonics education directly and immediately connected to current and emerging real world applications, with potential for societally meaningful, professionally satisfying and materially lucrative jobs for our students - all strong motivating factors for our students. The idea of a computer-controlled fully autonomous vehicle dates back as long as 1969 [3]. However, the race to actually assemble and test such a vehicle was truly opened by the 2004 Defense Advanced Research Projects Agency (DARPA) Grand Challenge. Though none of the 15 competing autonomous vehicles of 2004 were able to complete the 142 miles course through the Mojave Desert, a winner (Stanford's Stanley, Figure 1) did emerge at the subsequent 2005 DARPA competition. It was equipped with five

Fifteenth Conference on Education and Training in Optics and Photonics: ETOP 2019, edited by

Anne-Sophie Poulin-Girard, Joseph A. Shaw, Proc. of SPIE Vol. 11143, 111430C · C 2019

SPIE, ICO, IEEE, OSA · CCC code: 0277-786X/19/\$18 · doi: 10.1117/12.2523863 
SICK LMS-291 two-dimensional lidar units. At the same race the first version of the 64-lasers Velodyne lidar developed by the Hall brothers - today a mainstay of the lidar marketplace landscape - was introduced.
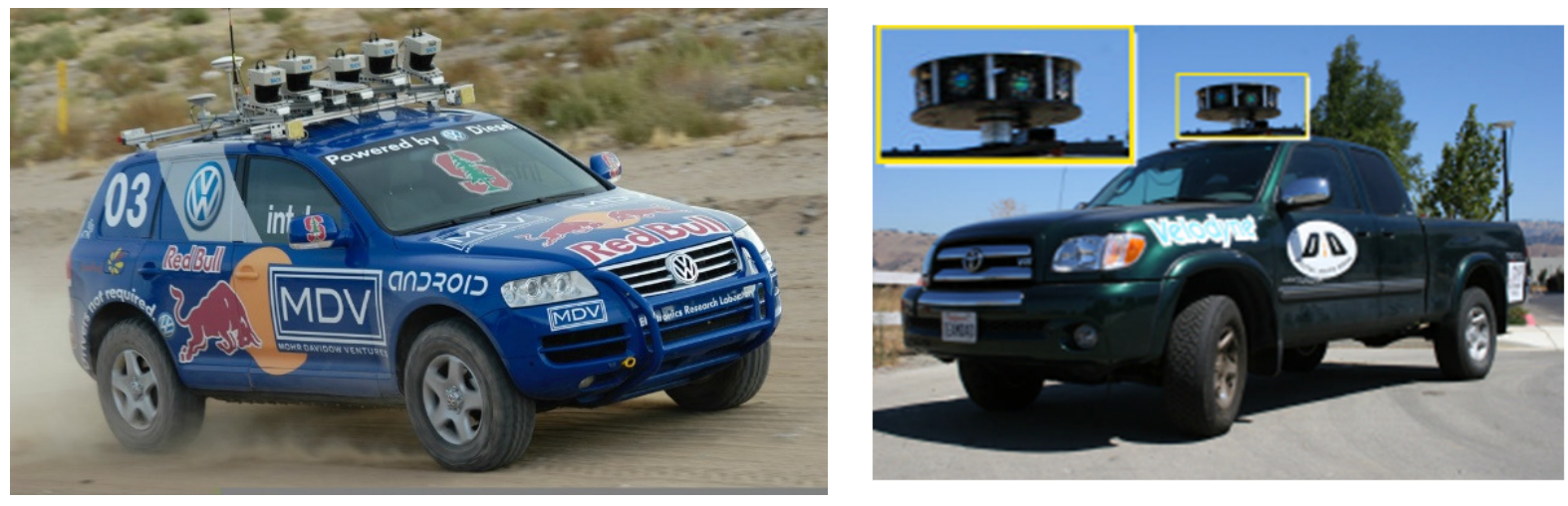

Figure 1. Stanley, the winner of the 2005 DARPA challenge equipped with five SICK LMS-291 2D LIDAR units and Hall brother's "Team DAD" truck equipped with their own 64-lasers spinning disc Velodyne 3D LIDAR (Source: DARPA)

Although the Hall brothers did not win the race, their $360^{\circ}$ spinning-disc lidar piqued the interest of other teams to such extent that, in 2007, five out of the six eventual winners of DARPA's last competition were actually using Velodyne lidar. In 2009, Google began developing in secret its self-driving car project, which later became its spin-off company Waymo, initially using the Velodyne lidar, but eventually developing its own. By the end of 2018, Google's autonomous car had driven more than 2 million miles. By 2014 all major name companies like GM, Ford, BMW, Mercedes, Nissan, Toyota, Hyundai, Volvo, Tesla, Uber and even (allegedly) Apple were working on their own self-driving car technologies. A new impetus to the autonomous vehicle race seems to have come in early 2016, brought about by a letter from the National Highway Traffic Safety Administration (NHTSA) in response to a request for legal clarifications by Google. The February 9, 2016 letter [4] essentially said that the computer running an autonomous vehicle could legally qualify as the "driver," with all the implied legal and technological consequences. The letter was soon followed by a flurry of company acquisitions and rounds of capital investments in suppliers of technology for the autonomous vehicle market space notably in companies delivering lidar solutions. The pace at which advances in this field are made keeps going up, facilitated in part by major advances in computing power and artificial intelligence (AI), which allow the integration (fusion) of the various sensors used by the autonomous vehicle. In this context, the market for lidar components, systems, integration platforms and applications has been sliced and diced in all possible ways over the past two years in a number of market research reports, with a significant focus on the fast-growing market segment of automotive LIDAR in particular. According to one such report by BIS Research [5], the automotive lidar market is estimated to grow from $\$ 353.0$ million in 2017 to $\$ 8.32$ billion by 2028 , at a combined annual growth rate of $29.6 \%$, and reach $\$ 44$ billion by 2050 [6]. This however pales in comparison to the broader societal impact forecasted by a Strategy Analytics report commissioned by Intel [7], which predicts a 7 Trillion economic impact by 2050 of the so-called passenger economy enabled by the deploying of autonomous vehicles.

\subsection{Sensors for autonomous vehicles}

LIDAR is only one of the four types of sensors that feed information into the AI-controlled integration platforms that eventually controls the vast majority of autonomous or semi-autonomous vehicles. This information eventually allows the construction of a comprehensive 3D map of the vehicle environment and a classification of the objects within it. The complete suite of 3D sensing technologies in use for autonomous vehicles includes:

- Cameras (B\&W and color) for passive visual sensing, working in tandem with sophisticated object detection algorithms, are used to collect a $360^{\circ}$ view of the local environmental context such as traffic signs and signals, and for lane departure warning.

- Radar, used for detection, localization and tracking (range and velocity) of objects using millimeter radio waves at 24,77 or $79 \mathrm{GHz}$. A long established mature technology available at affordable costs, radar performs very well in extreme weather conditions and at long distances (200+ meters). However, due to its use of radio waves (as opposed to light), it does not offer the angular and linear resolution of lidar. Long-range radar (LRR), having the longest scanning range of all sensors, allows for the detection of objects and hazards at high speeds and long 
distances (i.e. in highway driving), and is thus used for automatic distance control (adaptive cruise control) and emergency breaking. Short-range radar, on the other hand, is used for blind spot monitoring, lane-change assist, park assist and rear end collision warning. Essentially, short-range radar is intended to replace ultrasonic sensors.

- Ultrasonic sensor, serving the same purpose as (a substitute for) short-range radar

- LIDAR. Long-range lidar is used for detection, localization and identification/classification of objects at long distances (up $200 \mathrm{~m}$ ), such as for pedestrian identification, collision avoidance and emergency breaking. While its performance in poor weather conditions is worse than that of radar, lidar offers the advantage of very high angular and linear resolution due to its use of light waves. Short range lidar, on the other hand, is used to monitor the immediate surrounding of the vehicle (e.g. around the bumpers). Unlike short-range radar, however, shortrange lidar not only detects the presence of objects, but it also identifies them (e.g. a fire hydrant vs a child on a sidewalk).
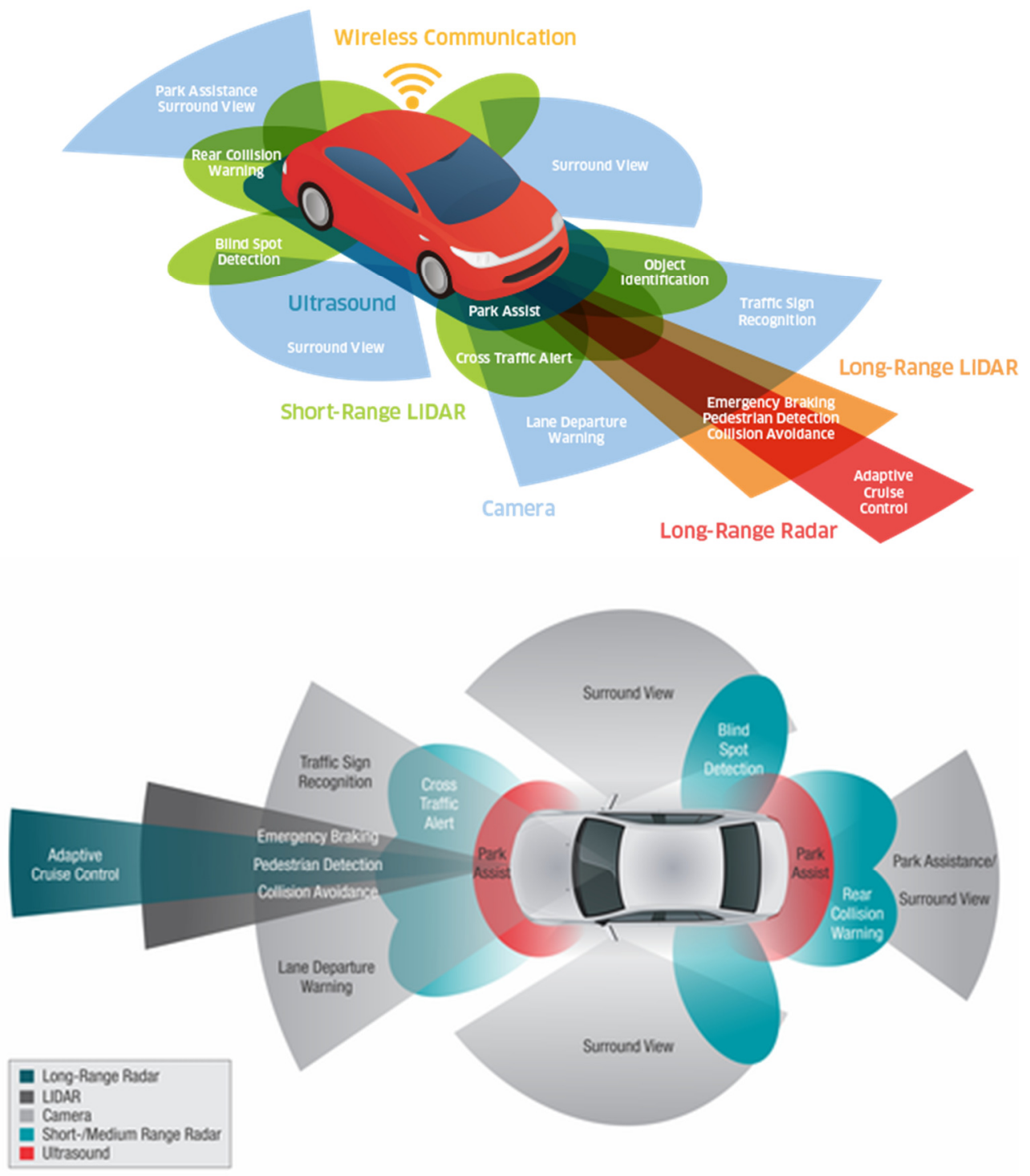

Figure 2. Two possible sensor-coverage diagrams (Source: Lumentum Holdings, Inc., https://www.lumentum.com/)

As the diagrams in Figure 2 reflect, the specific sensor combination and their coverage do vary from manufacturer to manufacturer, and the optimum combination is a matter of current debate. Nonetheless, with Tesla's notable exception, the need for a combination of cameras, radar and lidar is broadly accepted among autonomous driving solutions providers, as this combination provides both the complementarity and redundancy needed for safe operation. 


\section{AUTOMOTIVE LIDAR DRAWS HEAVILY ON OPTICS AND PHOTONICS}

\subsection{Basic LIDAR operating principle}

Scientists have used lasers to measure distances shortly after the advent of the laser in 1960. In 1969 the Laser Ranging Retroreflector was deployed on the surface of the Moon by the Apollo 11 Mission, allowing an experiment first proposed in 1962 by then Princeton University graduate student James Faller. During their historic moonwalk on July 20, 1969, Neil Armstrong and Buzz Aldrin deployed a 46-cm square laser retroreflector array containing 100 corner cube reflectors, based on Faller's design. Two weeks later, on August 1, 1969, a laser pulse aimed through the lens of the 3-meter telescope at California's Lick Observatory successfully hit the array for the first time. By measuring the time it took for the beam to perform the round trip (about 2.5 seconds) the distance between the Earth and the Moon was calculated with an uncertainty of \pm 25 centimeters [8]. Two years later, in 1971, the first space-borne lidar was launched aboard Apollo 15. Using a 694$\mathrm{nm}$ ruby laser operating at a rate of $0.05 \mathrm{~Hz}$, the so-called Apollo Laser Altimeter mapped the elevation profile of the Moon's surface for the first time. This opened the door to one of the most popular uses to this day of lidar, namely aerial mapping of large areas of land (Earth surface), with applications in agriculture, forestry and archeology.

The basic principle of lidar operation (similar for radar or sonar, for that matter) is illustrated in Figure 3a. A pulse of energy (wave packet) is launched from a source, reflected back by the target and detected by an appropriate sensor. By measuring the roundtrip travel time, one calculates the distance. The nature of the waves employed differentiates between SONAR (ultrasonic waves), RADAR (radio waves) and LIDAR (light waves). This type of lidar is known as time-of-flight lidar. The speed and wavelength of the specific waves employed determine the distances they travel in a given time, the divergence (spreading out) and scattering of the beam while it propagates through a medium, and eventually determines the usefulness of sonar, radar and lidar for specific applications, including in autonomous vehicles in terms of maximum range and resolution.
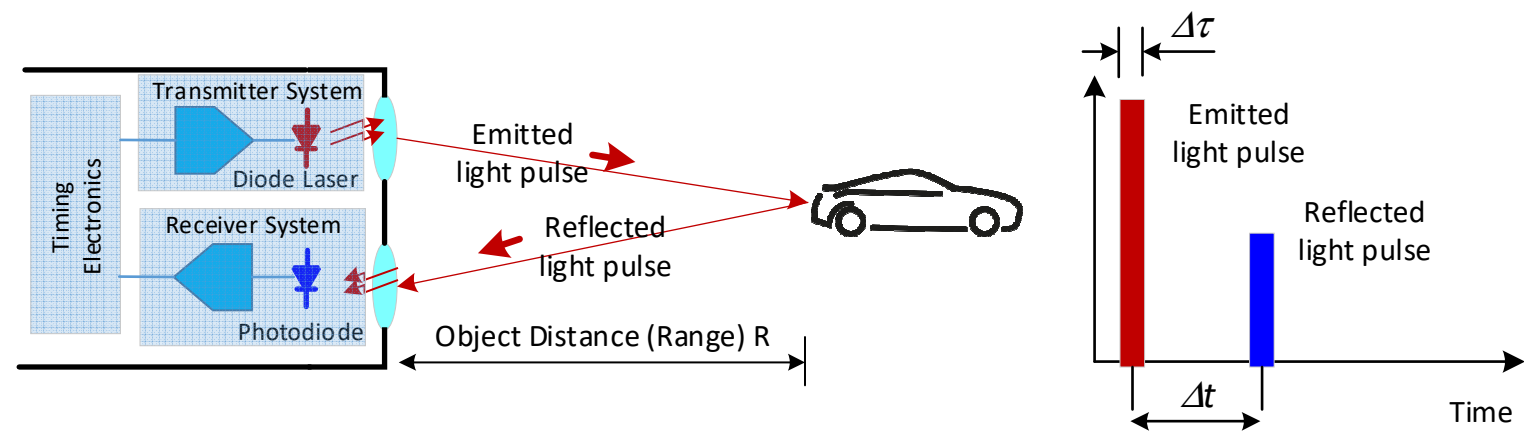

Figure 3. Basic lidar operating principle (a), and the timing diagram of the pulses (b).

The distance to the object (range) is then calculated as $R=\frac{v \cdot \Delta t}{2}$, where $v$ is the speed of the energy pulse and $\Delta t$ is the roundtrip travel time. In the case of lidar, a light pulse is employed, typically a very short laser pulse. Given the very high speed of light $\left(\cong 3 \times 10^{8} \mathrm{~m} / \mathrm{s}\right.$ in air, assuming an index of refraction of 1$)$, a target at $150 \mathrm{~m}$ results in a round trip of $\Delta t=$ $1 \mu s$. This extremely short time makes it possible to construct a 3D point-cloud image of the target by scanning the laser beam across the target in a raster pattern, measuring the individual travel time for each horizontal (azimuthal) and elevation angle of the laser beam, and calculating the corresponding distances. Figure 4 presents two color-coded point cloud images produced by the $1550 \mathrm{~nm}$ scanning-mirror(s) lidar manufactured by Luminar, a Palo Alto, CA based startup company. Assuming distances around $150 \mathrm{~m}$, at 1 microsecond roundtrip one can collect one million points per second. The density of the point cloud and thus the lateral (transverse) resolution of the image it produces depends of course on the range of the target and on the horizontal (azimuthal) and vertical angular fields of view to be covered. The distance (range) resolution, on the other hand, is determined by two factors, as illustrated in Figure 3b, namely the uncertainty in measuring the round trip time, $\delta(\Delta t)$, and the temporal spread of the laser pulse itself, $\delta \tau$, which results in a spatial length of the pulse of $\delta l=c \cdot \delta \tau$. For a target range resolution of $\delta R=5 \mathrm{~cm}$, both $\delta(\Delta t)$ and $\delta \tau$ need to be on the order of $0.3 \mathrm{~ns}$. Thus, time-of-flight lidar requires photodetectors and detection electronics with small time jitter - the main contributor to $\delta(\Delta t)$, and very short laser pulses (small $\delta \tau$ ). Of course the problem gets a bit more complicated in the case of a moving target or a moving lidar unit, as is the case for automotive lidar, which is a real time lidar. 

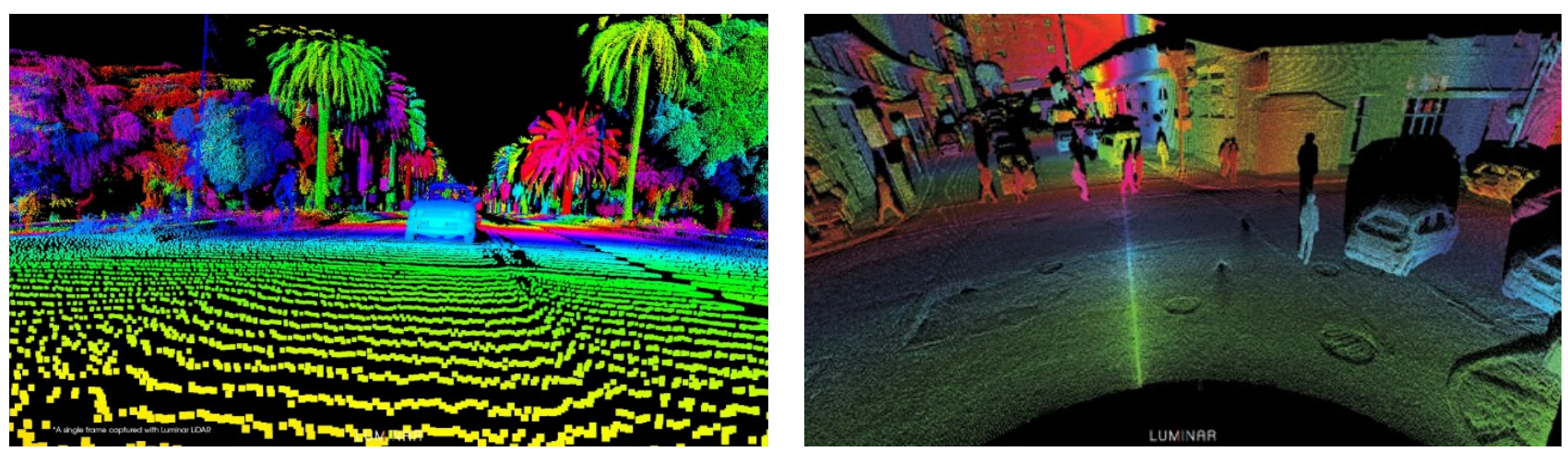

Figure 4. Single frame images produced by Luminar's LIDAR sensor (Courtesy of Luminar)

Although this section concerns itself with technical rather than pedagogical aspects of teaching lidar fundamentals, the connections to topics and discussions in any Waves, Optics or Photonics course is obvious. In particular, approaching this as a solution to an engineering problem rather than a pure Introduction to Optics problem has the immediate potential of shaping the discussion and grabbing the student attention, particularly when teaching optics to non-Physics or Optics majors. A typical, "boring" problem of relating distance, speed and time becomes more engaging when framed in the context of the density of point cloud allowed by a light beam at a given distance. Likewise, a problem of propagation of uncertainties acquires very real and critical meaning in the context of the range resolution of the lidar. In a similar fashion, the problem of wave diffraction by an aperture gets an immediate meaning in the discussion of the divergence of the laser and radar beams, which limits the angular resolution and renders radar incapable of resolving individual objects at large distances, but makes lidar very useful for object identification through a better angular resolution which translates into better transverse resolution. Indeed, basic wave physics dictates that the divergence of the wave beam is determined, through diffraction, by the ratio of the wavelength to the aperture diameter of the emitting antenna (radar) or lens/mirror (lidar).

\subsection{Emission, propagation and detection of light in LIDAR operation}

\subsubsection{Lasers and detectors for LIDAR}

As discussed in section 2.1, the operation of a time-of-flight lidar requires a pulsed laser capable of producing laser pulses a few nanoseconds long and with a high repetition rate. Wavelength, power, pulse length and repetition rate, as well as beam divergence are key parameters that impact the construction and performance of the lidar unit. Eventually, the selection of a given laser for a lidar unit is determined by the specific mode of lidar operation (more on this later) and by the performance, availability and cost of not only the laser itself, but also of the required photodetectors. The three most common currently used or explored wavelengths for automotive lidar are $905 \mathrm{~nm}, 940 \mathrm{~nm}$ and $1550 \mathrm{~nm}$, each with its own advantages and drawbacks. One consideration in lidar design is the presence of ambient light which can interfere with its operation. As such, an operating wavelength that corresponds to a local minimum in the solar spectrum at the surface of the Earth is preferable. The solar spectrum has such minima around $905 \mathrm{~nm}, 940 \mathrm{~nm}$ and $1550 \mathrm{~nm}$ caused by absorption by water vapor in the upper atmosphere. Of course, the same absorption can have a detrimental effect on the roundtrip propagation of the lidar laser beam itself. Nonetheless, $905 \mathrm{~nm}$ has long been the standard wavelength for range-finding lidar, and its use in the well-established and widely deployed Velodyne $360^{\circ}$ spinning lidar has consolidated its position on the market significantly. Also, low cost high power edge emitting pulsed diode lasers at $905 \mathrm{~nm}$ are readily available from companies like Lumentum, Hamamatsu, Osram and many others, and so are the necessary photodetectors (silicon photodiodes or photodiode arrays), since $905 \mathrm{~nm}$ is within the range of detection by silicon, and thus also compatible with CMOS detector array technology.

One drawback of $905 \mathrm{~nm}$ lasers is that they fall within the range of wavelengths that can penetrate through the front and the interior vitreous humor of the eye, and reach the sensitive retina. Thus safety rules limit the allowed power density that can be employed in lidar operation, and through that, they limit the $905 \mathrm{~nm}$ lidar range to within tens of meters to $100 \mathrm{~m}$. The $1550 \mathrm{~nm}$ wavelength offers a significant advantage in this respect, as it falls beyond the $\sim 1400 \mathrm{~nm}$ retinal hazard limit. Light beyond $1400 \mathrm{~nm}$ gets absorbed in the front layers of the eye (cornea, aqueous humor and the lens) mainly because of watery absorption, and does not reach the retina. Power levels as much as 10 times or even 40 times higher than at 905 $\mathrm{nm}$ can be used. Also, the number of $1550 \mathrm{~nm}$ photons to be detected at any power level is 1.7 times larger than that of $905 \mathrm{~nm}$ photons at the same power, and less sunlight reaches the ground at $1550 \mathrm{~nm}$ compared to $905 \mathrm{~nm}$. Because of all 
these, $1550 \mathrm{~nm}$ lidar can achieve longer range. Luminar, a lidar start-up company in Palo Alto, CA that has teamed up with Volvo, reports a range of more than $200 \mathrm{~m}$ at only $10 \%$ target reflectivity for its macroscopic-mirror mechanical scanning $1550 \mathrm{~nm}$ lidar. The use of the $1550 \mathrm{~nm}$ laser diodes as well as other components for fiber optics communications networks is also an advantage exploited in the development of coherent Frequency Modulated Continuous Wave (FMCW) lidar, discussed in section 2.3. Since this type of lidar requires continuous wave lasers with long coherence length, narrowlinewidth distributed Bragg reflector (DBR) diode lasers are used, like those supplied by Lumentum (Milpitas, CA) The downside of a $1550 \mathrm{~nm}$ lidar is the increased cost of the detector, as well as the lack of detector array offerings (for flash lidar), since more exotic materials like Ge, InGaAs, or InGaAsP detectors have to be used. The use of optimized detectors is paramount to the lidar performance, and another way to improve the range of a lidar unit. Because only a small fraction of the photons emitted by the laser make it back to the detector, selecting the right photodetectors with high detection sensitivity, high internal gain and low noise is critical. For $905 \mathrm{~nm}$ lidar, silicon avalanche photodiodes (APDs), singlephoton avalanche diodes and silicon photomultipliers (SiPMs) are popular detectors, each with its specific advantages and limitations. For $1550 \mathrm{~nm}$ lasers, mostly InGaAs photodiodes or avalanche photodiodes are used.

Other choices of lidar wavelength can be used as well. Aside from its $1550 \mathrm{~nm}$ DBR diode lasers, for example, Lumentum also offers $940 \mathrm{~nm}$ vertical cavity surface emitting lasers (VCSEL) optimized for high resolution flash lidar. Ouster, a multi-beam flash lidar start-up company in San Francisco, CA uses $850 \mathrm{~nm}$ VCSELs, a choice the company says runs counter to the "trend" of $905 \mathrm{~nm}, 940 \mathrm{~nm}$ or $1550 \mathrm{~nm}$, but one they claim they have turned to their advantage through a patented ambient light rejection approach that takes care of the higher solar background at $850 \mathrm{~nm}$ (10 times higher than at $940 \mathrm{~nm}$ and 3 times higher than at $1550 \mathrm{~nm}$ ). With that, the use of $850 \mathrm{~nm}$ laser offers better performance in humid weather due to lower water vapor absorption at $850 \mathrm{~nm}$ (but not through rain or fog). The $850 \mathrm{~nm}$ wavelength also results in improved sensitivity (by a factor of 2, when compared to $905 \mathrm{~nm}$ ) of the silicon CMOS detector array used in the flash lidar, to the point where (per company claim) "the lidar is the camera." The company's proprietary software for its OS-1 lidar unit allows the CMOS array to collect ambient imagery in addition to lidar images.

\subsubsection{Propagation of light in LIDAR operation}

Three fundamental optics phenomena bear heavily on the performance of a lidar system: absorption, scattering and reflection of light. In contrast to radar, lidar has only limited performance in poor weather conditions (rain, snow, heavy fog). This is due mainly to absorption of light by water and to atmospheric scattering of light out of the directional laser beam, thus reducing the photon flux available for reflection by the target and eventually for detection by the lidar unit. The reflection of light, on the other hand, is what allows the lidar to detect the world around it. The reflections used for constructing the 3D point cloud images are obviously mostly the diffuse reflections from the various points of the target scene, but both diffuse and specular reflections contribute and affect the performance of the system.

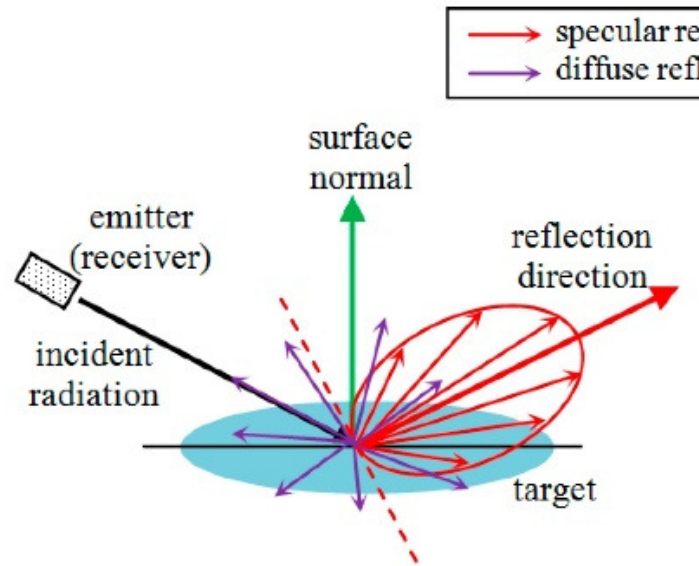

(a)

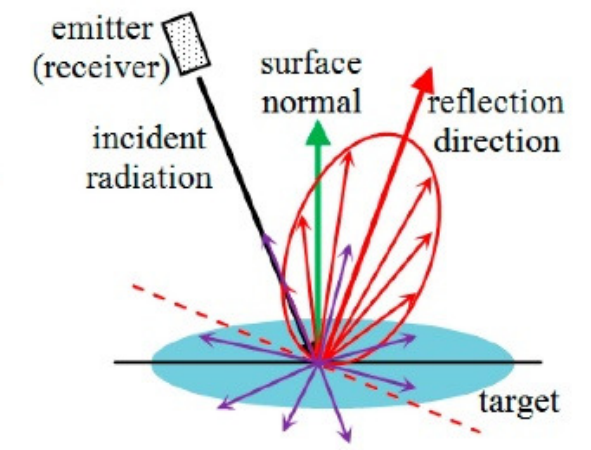

(b)

Figure 5. Diffuse and specular reflection by a surface. At large incidence angles, only diffuse reflections reaches the receiver of the LIDAR unit (a); at smaller incidence angles, both diffuse and specular reflection reach the receiver (b) [Reference 9]

A common student question and misunderstanding in discussing the basic principle of LIDAR as presented in Figure 3 is "If the light source and photodetector are in fixed relation with each other inside the LIDAR unit, how come light from the 
laser can be reflected back into the detector from multiple points of the target scene, that could be very far apart from each other. Specular reflection would dictate that only one point on the target could be seen." This opens the discussion of specular vs diffuse (surface vs body) reflection, which introduces the concept of bidirectional reflectance distribution function (BDRF) of a surface and, in that context, the concept of a Lambertian surface.
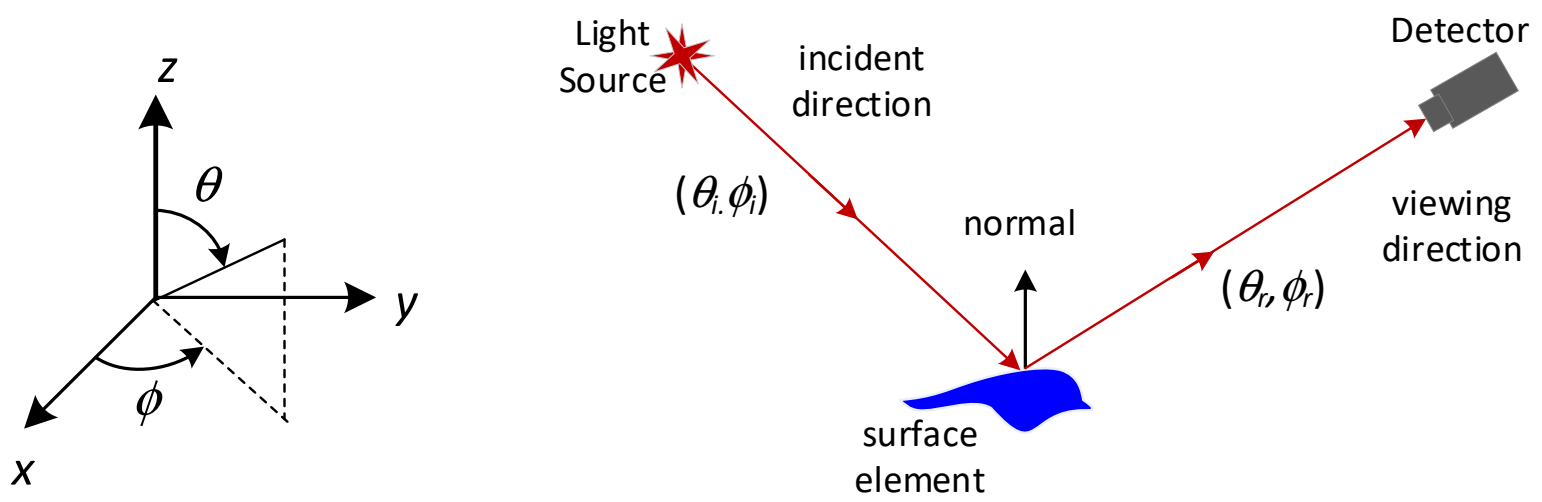

$$
\begin{gathered}
B R D F=f\left(\theta_{i}, \phi_{i}, \theta_{r}, \phi_{r}\right)=\frac{L^{\text {surface }}\left(\theta_{r}, \phi_{r}\right)}{E^{\text {surface }}\left(\theta_{i}, \phi_{i}\right)} \\
E^{\text {surface }}\left(\theta_{i}, \phi_{i}\right)=\text { Incident irradiance at surface in direction }\left(\theta_{i}, \phi_{i}\right) \\
L^{\text {surface }}\left(\theta_{r}, \phi_{r}\right)=\text { Radiance of the surface in direction }\left(\theta_{i}, \phi_{i}\right)
\end{gathered}
$$

Figure 6. Reflection of light by a surface is described by the bidirectional reflectance distribution function, BDRF.

In the case of a Lambertian surface (ideal diffusion surface), the radiance of the surface is isotropic and the BRDF of the surface is a mere constant:

$$
B R D F_{\text {Lambertian }}=f\left(\theta_{i}, \phi_{i} ; \theta_{r}, \phi_{r}\right)=\frac{\rho}{\pi}
$$

where $\rho$ is the reflectance (reflectivity) of the surface. This shows that the brightness of a Lambertian surface appears uniform from any direction of view. The end goal of this discussion is an estimation of power/photon budget available for detection by the lidar sensor. The reflectance of the different elements in a real-life target scene is one of the wildest cards in the operation of a lidar unit with huge impact on the detection range and implications about the way the range of any particular lidar units is quoted in the unit specifications. In the process of constructing the 3D point-cloud image of the vehicle surroundings, the lidar will scan over vehicles and static surrounding objects (some highly reflective, some not), highly reflective license plates and road signs, lane and road-side marking retroreflectors and others, that could each flood (saturate) the detector. At the same time other surfaces and objects like the road pavement, car tires, trees without foliage, etc. typically have low reflectance. The reflectance of the target and the desired detection range can impose rather stringent demands on the required performance of the photodetector(s) in the lidar unit. Assuming an ideal Lambertian surface, the power $P_{r}$ received by the detector inside the lidar unit can be expressed by an equation of the form:

$$
P_{r}=\frac{\rho P_{t} D_{r}^{2} \eta_{a t m} \eta_{s y s}}{4 R^{2}} \cos \Theta_{i}
$$

$P_{r}$ is thus proportional to the surface reflectance $\rho$, to the laser output power $\left(P_{t}\right)$, to the receiver area $D_{r}^{2}$ and the cosine of the incidence angle. Notably, the power reaching the detector goes down with the square of the range, $R^{2}$, and is affected by losses in air (through absorption and scattering, $\left.\eta_{a t m}\right)$ and losses within the system itself $\left(\eta_{s y s}\right)$.

The problem of ambient light - which can result in a degraded signal-to-noise ratio of the detected signal - and that of the losses within the lidar unit itself brings about another fundamental light phenomenon and major component of any optics or photonics course: interference of light, with focus on thin film interference, narrow band-pass filters and antireflection 
coatings. Indeed, to minimize the reflective losses of the useful signal at the operation wavelength while also maximizing ambient light rejection, the lidar unit must incorporate appropriately coated optics with multiple capabilities. This includes narrow band-pass filters at the lidar operating wavelength in front of the detector, and appropriately coated external windows for the entire lidar unit, such as those developed by Viavi Solutions of Milpitas, CA, a company with established expertise in optical coatings. The problem of (multiple) reflections gets even more complicated when the lidar unit is integrated with other components of the vehicle. For example, Magneti Marelli, an automotive supplier company with strong presence in the automotive lighting market has introduced the concept of Smart Corner ${ }^{T M}$ which aims for the integration of all sensors (cameras, radar and lidar) into the headlight and taillight fixtures of the vehicle. This approach, while desirable and attractive from the vehicle design aesthetics point of view, potentially creates additional light reflection problems that have to be identified, quantified and addressed.

\subsection{Types of LIDAR}

The type of lidar described in Section 2.1 is referred to as time-of-flight lidar and can itself take several different forms, as reflected in the LIDAR classification below:

* Time of flight lidar

- Scanning lidar

- Mechanical scanning lidar

- Solid state scanning lidar

- Microelectromechanical systems (MEMS) mirror lidar

- Optical Phase Arrays (OPA) lidar

- Flash lidar

- 2D (line) flash lidar

- 3D (whole scene) flash lidar

* Coherent Frequency Modulated Continuous Wave (FMCW) lidar

* Other (spectral scanning, micropolarizer camera)

As one might expect, each lidar type comes with its own advantages, downsides and challenges yet to be overcome.

\subsubsection{Time of flight scanning LIDAR}

The principle of operation of time of flight scanning lidar was discussed in detail in Section 2.1. Such a lidar measures the roundtrip travel time of photons from laser to target and back and converts it to distance. Using the distances so measured, the lidar constructs the 3D point cloud map of the objects encountered within the target solid angle one point at a time (single laser lidar) or one line of points at a time (multiple lasers lidar). The most widely used scanning lidar, with the longest history and most miles of autonomous driving field trials on multiple car makes and models is the Velodyne rotating scanner HDL-64E. Figure 7a through c presents the Velodyne spinning lidar models available in 16, 64 and 128 channels.

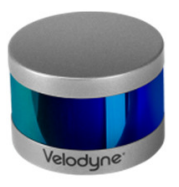

(a)

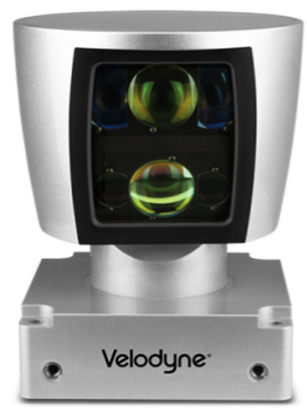

(b)

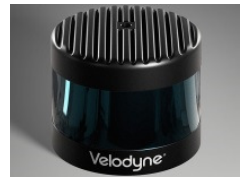

(c)

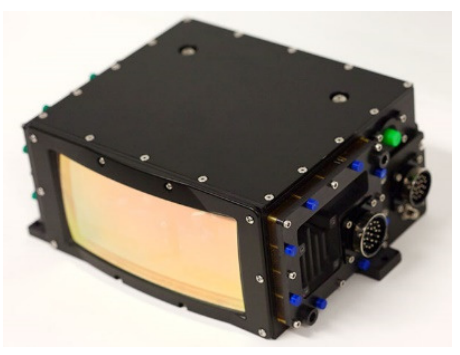

(d)

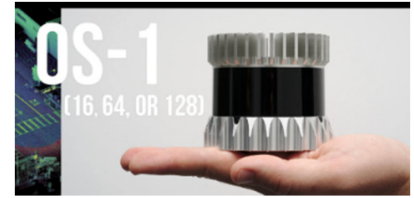

(e)

Figure 7. Velodyne's $360^{\circ}$, $905 \mathrm{~nm}$ spinning lidar with 16, 64 and 128 lasers respectively) (a), (b) and (c); Luminar's 1550 $\mathrm{nm}, 200 \mathrm{~m}$ range macroscopic scanning mirror lidar (d); and Ouster's OS-1 $850 \mathrm{~nm}$ flash lidar/CMOS camera (e)

The HDL-64E uses a spinning set of 64 stacked laser-detector pairs which scan simultaneously 64 separate horizontal planes covering a total vertical angle of $26.9^{\circ}$. Each laser has a dedicated avalanche photodiode detector (APD). This results in $\sim 0.4^{\circ}$ horizontal (azimuth) angular resolution. The stack spins full $360^{\circ}$ at $10-30 \mathrm{~Hz}$ resulting in a horizontal 
angular resolution of $0.08^{\circ}$. The 64 sources are $905-\mathrm{nm}$ laser diode sources which emit 5 -ns $60 \mathrm{~W}$ pulses which allow a 120 -m range with a $\pm 2 \mathrm{~cm}$ resolution at 0.8 target reflectance (e.g. cars, foliage) and a $50 \mathrm{~m}$ range at 0.1 target reflectance (pavement).

The moving parts and complexity of a mechanical scanning lidar like Velodyne and Luminar result in high cost even at large production volumes, and represent a failure risk in mechanically and environmentally rough driving environments. Because of that, many experts believe the key to making lidar affordable and reliable for autonomous vehicles is to move toward solid-state designs with no moving parts. That obviously requires some mechanism for steering a laser beam in different directions without mechanically moving the laser or any macroscopic mirrors. MEMS scanning mirrors and optical phased arrays (OPA) are the two most commonly investigated approaches to solid state scanning lidar. In the case of MEMS scanning mirrors, though technically still a mechanically scanned system, the miniature scale of the scanning mirror, small inertia and high resonant frequency make it more robust to harsh mechanical and temperature environments while the microfabrication approach is suitable for scaling down price through large volume production. Owing to existing applications in other areas (like optical switches for fiber optics communications and microprojectors) the MEMS microfabrication technology using silicon is mature and can produce light-weight, compact and low power dual-axis (tiptilt) MEMS mirrors with diameters from a fraction of a $\mathrm{mm}$ up $9 \mathrm{~mm}$, like the ones produced by Mirorcle Technologies of Richmond, CA. These aluminum- or gold- coated silicon mirrors can handle the variety of laser wavelengths and powers that are used in most lidar systems. The low moment of inertia allows the mirror to perform a two-dimensional scan over the entire field of view in a fraction of a second, an advantage for the required real-time operation of a lidar unit. Another advantage is that a MEMS lidar scanner can dynamically adjust its scan pattern to a finer grain (smaller angular step) in order to focus on objects of particular interest. Likewise, compared to existing large-scale galvanometer optical scanners, MEMS mirror scanners use orders of magnitude less driving power. Unlike a spinning lidar, however, a MEMS mirror lidar will have a limited angular field of view (horizontal and vertical), so multiple units are needed to generate a complete $360^{\circ}$ view. Also because of the small dimension (aperture) of the mirror, stronger diffraction occurs resulting in larger divergence of the projected beam. This in turn translates into shorter lidar range. Two startup companies working on MEMS-based lidar solutions are Innoviz (backed by Delphi) and Innoluce (backed by Infineon).

The third type of scanning lidar and the one with a true "no-moving parts" design is the optical phased array (OPA) lidar, which promises excellent reliability and is thus very attractive for automotive lidar. At this point, however, this is the least developed technology, not yet deployed or tested in autonomous driving on any meaningful scale, but one with great promise for the future nonetheless. While steering in two dimensions is possible, many current typical OPA will allow steering of a laser beam in one dimension (one plane) only. The 1D OPA consists of a number of optical elements (antennas) arranged in a one dimensional array, as presented schematically in Figure 8a.

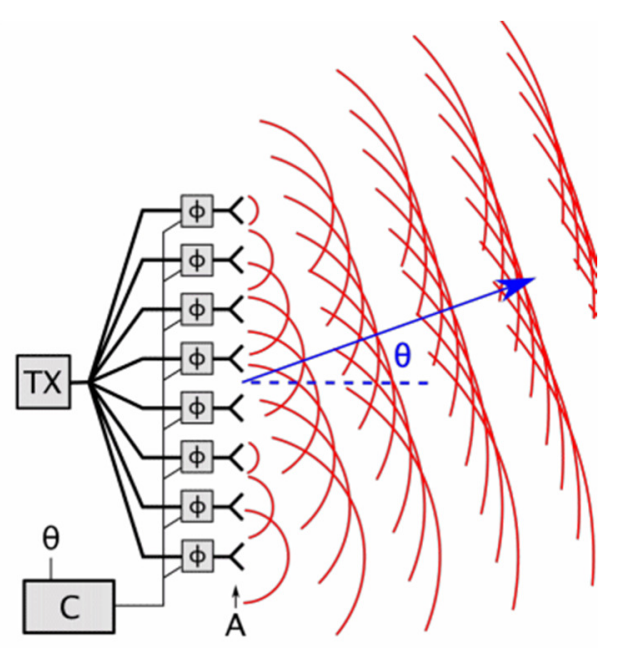

(a)

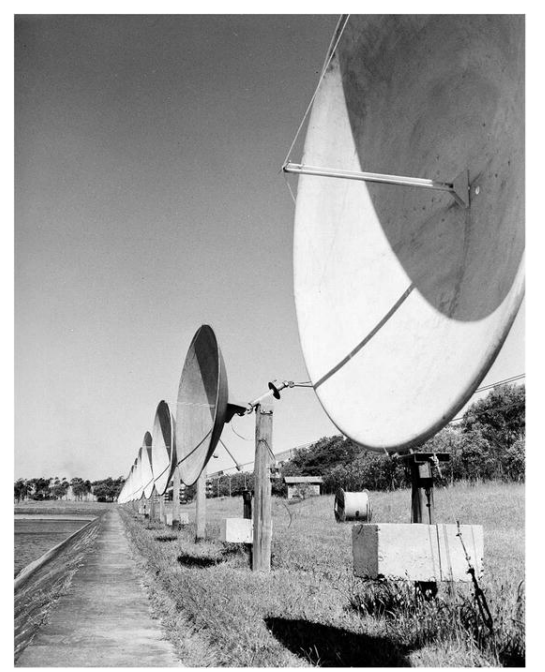

(b)

Figure 8. Schematic of the steering operation principle of a 1D phased array (from Wikipedia) (a), and its implementation in the 1951 32-element solar grating array (radio astronomy telescope0 designed and constructed by Chris Christiansen at Potts Hill, Australia. (CSIRO Radio Astronomy Historical Photographic Archives B26) 
When illuminated with the coherent light from a laser, the elements in the array act as optical antennas that re-emit the incident light. By controlling the phase and amplitude of the re-emitted light from each element of the array (e.g. introducing a constant phase shift between consecutive reemitted waves), one controls the direction of the far-field maxima of interference, and thus can steer the beam to any particular direction in one plane. Changing the value of the phase shift changes the direction. The astute reader will recognize in the above description the textbook introduction to the multibeam interference and eventually diffraction (see Optics by Hecht, for example), and its analogy with the beam steering performed in radio astronomy. A classic example in this respect is that of the Chris Christiansen designed radio telescope of 1951 [10] presented in Figure 8b and discussed briefly in the optics textbook by Hecht.

To steer the beam in the second dimension, OPA scanner systems typically use a grating or a prism, changing the direction of light in the second direction based on its wavelength. This automatically implies the use of a tunable laser to allow full steering in two dimensions. A two-dimensional OPA, fully integrated at the chip level with the tunable laser was reported by researchers at University of California Santa Barbara in 2015 [11]. According to the authors, the paper presents "the first fully integrated two-dimensional steerable laser chip on a hybrid III-V/silicon platform.....This chip includes 2 tunable lasers, 34 amplifiers, and 32 photodiodes utilizing III-V material, as well as 32 phase shifters, 31 MMI couplers and a graded index (GRIN) lens in the silicon-on insulator (SOI) layer. These combine wavelength tuning with an optical phased array to accomplish 2D beam-steering without the need for moving parts or an external laser." The optical phased array in this case consists of 32 micro-structured solid state waveguides which act as phase shifters, a technology widely used in the fiber optics industry for the manufacturing of Array Waveguide Gratings (AWG)-based dense wavelength division multiplexing (DWDM) multiplexers and demultiplexer modules. Unfortunately, at this time, high light losses in the various components of a solid state waveguides optical phased arrays restricts power and thus the usable lidar range. Quanergy is one startup company reportedly working on phased-array lidar, and so is Strobe, acquired by General Motors (GM).

A 2D scanner operating in reflected light by using liquid crystals phase modulators is being developed by Lumotive [12], a Seattle-based startup company funded by Bill Gates of Microsoft. By using a semiconductor chip having a reflective electronically controllable "liquid crystal metasurface" the device is claimed to be able to steer an incident laser beam in any direction, in any sequence. While liquid crystals are common for large scale phase modulators, they are generally considered too slow for the real-time demands of an automotive lidar system. Nonetheless, the large window of the device $(25 \mathrm{~mm} \times 25 \mathrm{~mm})$ does minimize diffraction and ensures small divergence of the outgoing laser beam.

\subsubsection{Time of flight flash LIDAR}

By contrast to a scanning lidar, a flash lidar is a truly "no-moving-parts" solid-state lidar that disposes of the scanning process altogether, and operates more like a flash camera. A laser beam is expanded to illuminate the entire scene, in a series of 2D flashes or 3D flashes. The time of flight point cloud is thus constructed either one horizontal plane at a time or the entire 3D point cloud in one flash, as opposed to the point-by-point construction in a scanning lidar. This clearly presents the advantage of eliminating timing issues raised by rapidly changing target scenes due to either motion of the target or motion of the lidar unit itself. The region illuminated by the laser flashes matches the field of view of the detector. The detector is a special CMOS array or some other 1D (linear) or 2D array of avalanche photodiodes (APDs) located at the focal plane of the detection optics. In effect, the target scene is divided into an array of scene elements (rectangles), each scene element being imaged onto a particular sensing element of the detector array, like in a digital camera. Unlike a camera though, each sensing element (APD) in the array measures the time of flight - rather than light intensity - to the scene element imaged on that particular sensing element. The transverse resolution of a time of flight flash lidar is thus limited by the total number of sensing elements (pixels) of the detector array. This imposes two conflicting demands on the detector array. Reducing the pixel size increases the total number of imaging pixels for a given size of the detector array and thus increases the transverse resolution of the lidar unit. At the same time, however, reducing the pixel size reduces the number of photons captured by that pixel and thus negatively impacts the signal to noise ratio. Because of laser power limitations and because of the laser beam being expanded to cover either the entire scene or at least one horizontal plane at a time, the effective range of a flash lidar is usually limited to a few tens of meters. One way to increase the power density and the range is to increase the number of laser beams emitted in one flash and/or improve the detection limit of the sensing elements in the array. To this end, VCSEL laser diode arrays can be employed, combined with single photon avalanche diodes (SPAD) CMOS arrays, like in the case of Ouster's OS-1 $850 \mathrm{~nm}$ flash lidar. A number of companies are developing flash lidar units. Figure 9a presents the core elements of a flash lidar sensor developed by LeddarTech, a lidar solutions company in Quebec City, while Figure $9 \mathrm{~b}$ presents a completely packaged unit of the Guardian $^{\mathrm{TM}}$ flash lidar sensor developed by Phantom Intelligence, also of Quebec City. Figure 10 shows how the target scene is segmented by the flash lidar into scene elements projected onto the sensing elements of the detector array. 


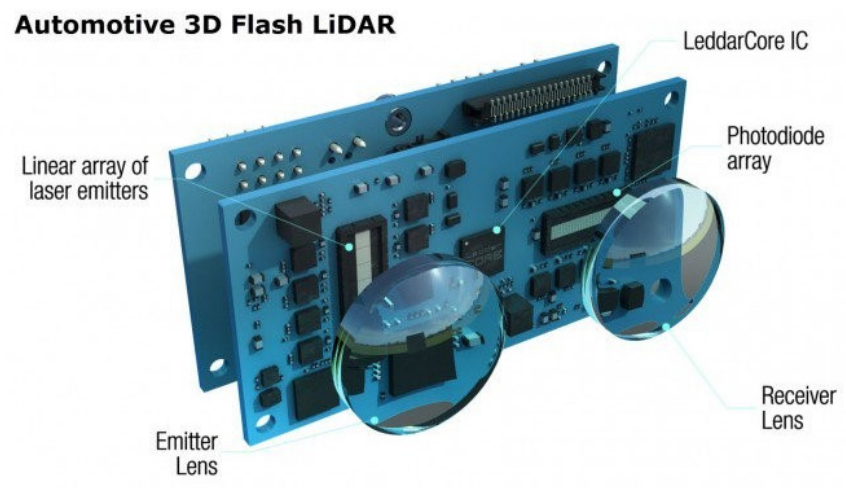

(a)

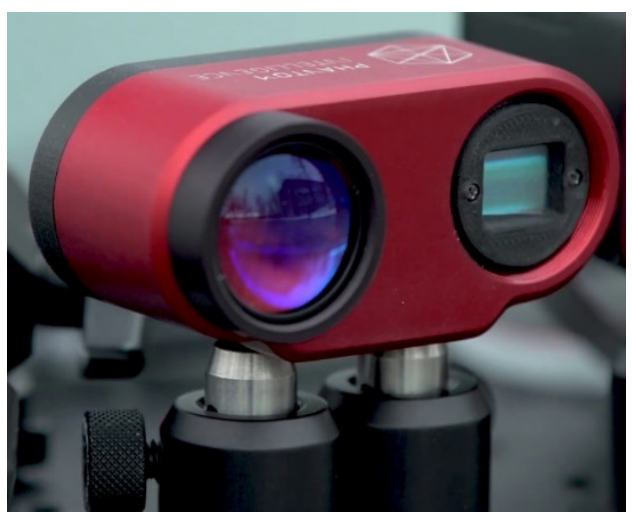

(b)

Figure 9. The core elements of a flash lidar unit (courtesy of LeddarTech) (a), and a complete Guardian ${ }^{\mathrm{TM}}$ flash lidar unit by Phantom Intelligence (b)

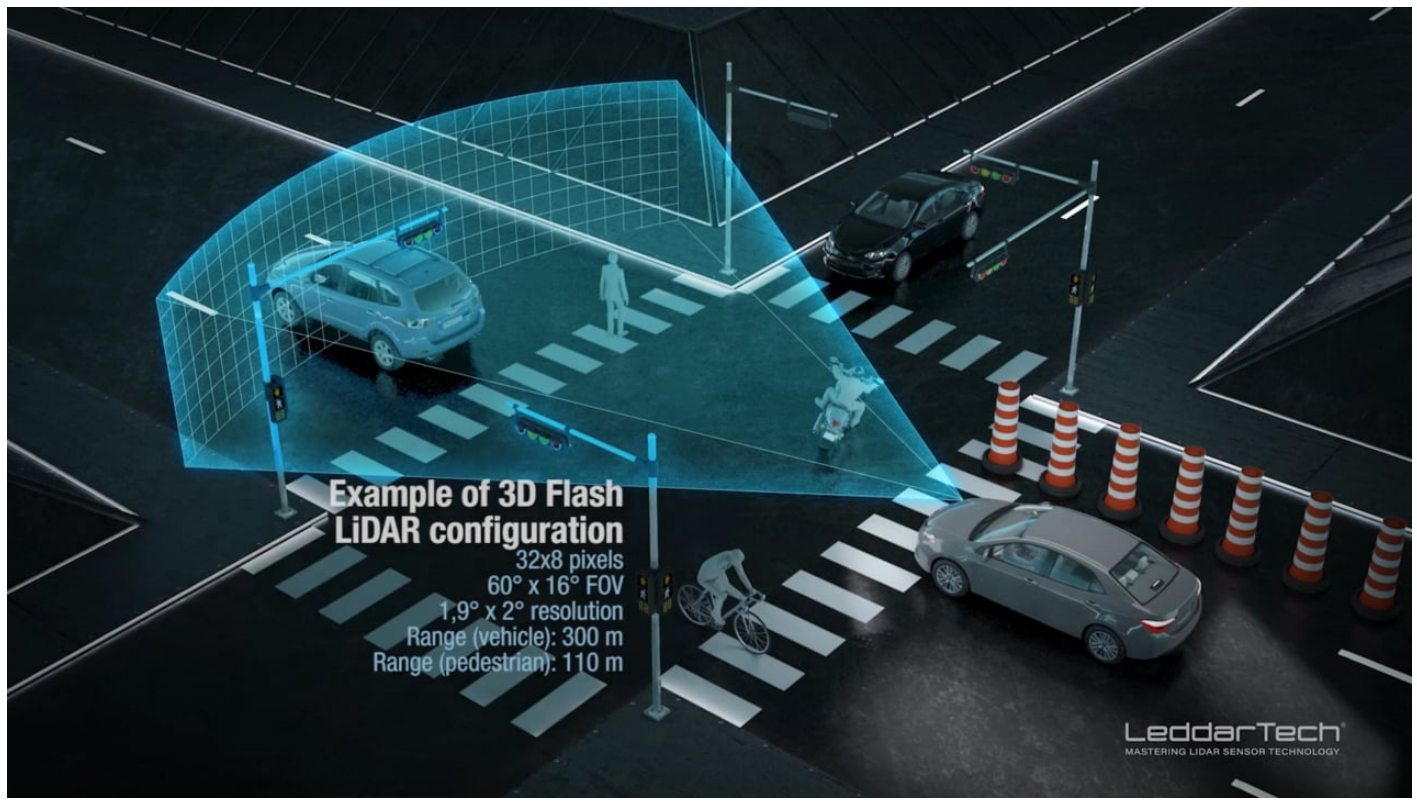

Figure 10. A target scene is segmented by the 3D flash lidar into scene elements (courtesy of LeddarTech)

\subsubsection{Coherent frequency modulated continuous wave (FMCW) lidar}

The coherent FMCW lidar is a more complex type of lidar, one that allows the simultaneous detection of not only the distance to the detected object, but also the velocity. The operation of a FMCW lidar is very similar to that of FMCW radar, also referred to as chirped radar. In chirped radar, the antenna continuously emits radio waves whose frequency is linearly increased from a base frequency $f_{0}$ to a maximum frequency $f_{\max }$ ("upchirp") over some period T, followed by a decrease in frequency from $f_{\text {max }}$ back to $f_{0}$ over the same period T ("downchirp") (Figure 11a). Assume the wave emitted at $t=0$ encounters an object located at some range $\mathrm{R}$ moving at some radial velocity $v_{r}$. After a time $\Delta t=2 R / c$ the back reflected wave reaches the transmitter-receiver, where it interferes with the wave emitted at that instant. The received wave will have a different frequency than the wave emitted at that instant due to two factors: the round-trip travel time $\Delta t$ determined by the range $\mathrm{R}$ of the object and the Doppler shift $\Delta f_{D}$ due to the wave being reflected from an object moving at relative velocity $v$. The interference of the two waves will produce a beats frequency $f_{B 1}$ on the upchirp side of the frequency modulation and a beats frequency $f_{B 2}$ on the downchirp side of the frequency modulation, as seen in Figure $11 \mathrm{~b}$. By measuring the two beat frequencies one can determine simultaneously both range and radial velocity of the object. 


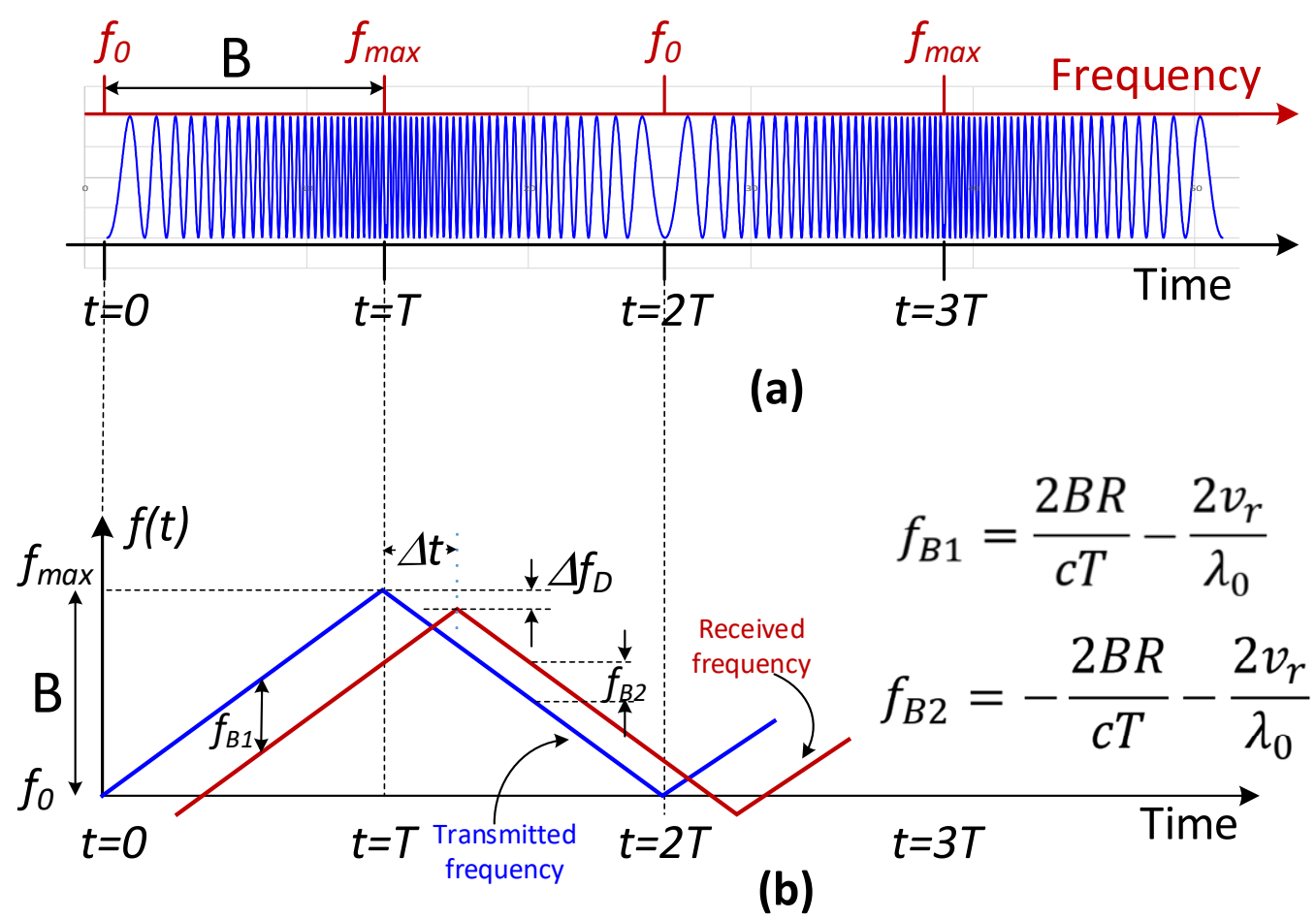

Figure 11. Linear chirp modulation of a signal (a), and the beat frequencies it produces through interference between the transmitted and reflected signal $(\mathrm{b}\}$

Inspired by chirped radar, one can devise a chirp-modulated lidar. One way to achieve this is by using a tunable laser to modulate the frequency of the laser itself (i.e. the carrier frequency). Another way is to use a fixed-wavelength laser and chirp-modulate its intensity. The above discussion of chirp-modulated carrier radar wave applies equally (including the Doppler shift) to the modulating envelope of (chirped modulated) laser intensity. To this end, the intensity-modulated returned light is detected by a photodetector to recover the modulation frequency and mix it with the frequency of the local oscillator allowing the measurement of the beat frequencies and the calculation of range and velocity of the object. Aside from being able to detect both distance and velocity of the target, FMCW lidar has also the advantage of being immune to a large extent to ambient light like sunlight or the laser beams of other lidar units on the road, a potential differentiating advantage compared to the simpler time of flight type pf lidar. At the same time, the approach has its own limitations and downsides. For one, the more complex approach of FMCW lidar requires more computational power to generate the 3D point cloud, which is now a point cloud of both distances and velocities. Also, the accuracy of the measurement depends very much on the linearity of the chirp ramp. The approach also imposes more stringent requirements on the laser source, as narrow linewidth long coherence lasers are required, often at $1550 \mathrm{~nm}$. Distributed Bragg Reflector (DBR) diode lasers are commonly employed, and fiber lasers or solid state lasers may be used as well. Two of the companies developing FMCW lidar for automotive applications are Blackmore of Bozeman, MT and Strobe of Pasadena, CA (owned by GM). The approach taken by both companies point toward full integration at the chip level. Blackmore's approach seems extremely promising. Per company statements, Blackmore's optical layer is built on standard optical fiber communications components, a major advantage that leverages decades of development in optical fiber communication and allows Blackmore to integrate designs to chip-scale lidar. Blackmore aims to combine this chip-scale lidar engine with solid-state scanners in silicon photonics to enable the mass manufacture of completely solid-state lidar sensors. The performance promised by this technology is impressive: a lidar sensor with single-photon sensitivity limited only by quantum noise capable of seeing through rain, snow and dust, but one with an extremely high dynamic range at the same time. Blackmore's multi-beam Doppler lidar sensor is said to deliver instantaneous velocity and range data beyond an impressive 450 meters. The system supports a 120 x 30-degree field of view, velocity measurements with accuracy down to 0.1 meters/second on objects moving up to $150 \mathrm{~m} / \mathrm{s}(335 \mathrm{mph})$, and measurement rates in excess of 2.4 million points/second. 


\section{AN OPPORTUNITY TO TEACH OPTICS AND PHOTONICS VIA LIDAR}

An automotive lidar system is a complex combination of opto-mechanics, passive optics and active photonics and optoelectronics components controlled by a multi-sensor integration platform running sophisticated image processing and artificial intelligence algorithms. In an ideal world, the output of such a system is a real time high resolution $3 \mathrm{D}$ point cloud image of the vehicle environment. In its more complex form (FMCW Doppler lidar) this point cloud image is a 3D map of both positions and velocities of the objects in the vehicle environment. As clearly apparent from the discussions in the previous sections, to teach a Fundamentals of LIDAR course is to teach at least a survey course in Optics and Photonics. Indeed, all fundamental optics and photonics phenomena and devices are well represented and central to the operation and performance of a lidar system. In effect, a course addressing lidar fundamentals and designs is an excursion through the entire ecosystem of optics and photonics phenomena, techniques and devices. A non-exhaustive list of optics topics that are well represented and central to the operation of the large variety of established or emerging automotive lidar designs includes

- light propagation and scattering

- diffraction of light by an aperture

- specular and diffuse reflection

- polarization by reflection and the Fresnel equations

- refraction by a prism, anamorphic prisms

- diffraction gratings

- $\quad$ high reflection and antireflection thin film coatings

- multiple beam interference and optical phased arrays

- chirp modulation of a signal, Doppler effect and beats

At the same time, the problem of laser light generation and detection is equally central to the operation of a LIDAR unit, and will expose the students to topics on active photonics devices like:

- $\quad$ side emitting laser diodes

- $\quad$ fixed wavelength and tunable vertical cavity surface emitting lasers (VCSEL)

- fiber lasers

- microelectromechanical systems (MEMS) devices (mirrors)

- $\quad$ optical phased arrays

- liquid crystals phase modulators

- $\quad$ photodiodes, avalanche photodiodes, single-photon detectors

- $\quad$ digital imaging sensors (CMOS detector arrays, CCD devices)

In addition, lidar designs that leverage established technologies employed in fiber optics communications offer the opportunity for at least limited discussions on such topics as well.

The race to a fully autonomous vehicle has by now migrated from the research labs to garages and factory floors, and from dedicated proving grounds to the city streets and neighborhoods. It is currently unfolding on a global scale and only picking up the pace. It is attracting capital investments and creating exciting new jobs that require skills across traditional science and engineering fields. At Kettering University, a school with tradition and history in automotive technology, a significant fraction of the student body pursues degrees in Mechanical Engineering, Electrical and Computer Engineering, and Computer Science. Many of these students may not have an interest in the field of optics and photonics as an end in itself, but are very interested in autonomous vehicle technology related careers. Kettering's participation in the intercollegiate AutoDrive challenge competition has only elevated this interest. One cannot overstate the intrinsic motivation factor represented by the sense of purpose ours students have for being involved in an engineering endeavor of the scale and global reach the autonomous vehicle market has ignited. This presents us with both a challenge and an excellent opportunity to expand our target audience for optics and photonics education by developing course modules addressing the principles of lidar operation and design. Such modules can be integrated as problems and examples of practical applications in a traditional optics and photonics course, can be offered as stand-alone units, or can be integrated in a teamtaught course on autonomous automotive technologies. Moreover, the discussion on autonomous vehicles has permeated more than only the technical segments of the academia and economic activity, and through its long-reaching societal implications has made the broader public a direct stakeholder in this endeavor. This again creates excellent opportunities 
for outreach activities that will engage the broader public and educate it about the technical aspects, career opportunities and the societal impact of optics and photonics solutions.

\section{CONCLUSIONS AND FUTURE WORK}

Even at the most general and superficial survey of lidar technology, types of lidar, their construction and principles of operation, it becomes quickly evident that to discuss lidar is to discuss optics and photonics. As optics and photonics professionals at Kettering, we see this as an opportunity to extend our reach and teach optics and photonics (in disguise) to a segment of our student population not otherwise interested in a traditional optics and photonics course. The intrinsic motivation factor for learning is significant and brought about by the sense of purpose our students have by becoming a part of the global autonomous vehicle challenge. The first step is for us - the educators - to educate ourselves about the established and emerging approaches to lidar, their underlying fundamental optics principles, and the advantages and challenges they pose. The first Velodyne automotive lidar units (64 or 128 lasers) were available at the prohibitive cost of about $\$ 75,000$ per unit. The current cost of a 16 -channel Velodyne scanning laser is about $\$ 7,900$. The target price for solid state lidar units - when volume produced for the automotive industry - is $\$ 100$ and below, and they should become largely available within the next two to three years. Some lidar sensors are already available for around $\$ 250$. By contrast, the speed of Light experimental setup currently available from PASCO costs $\$ 5,900$. This is not only an opportunity for teaching optics and photonics, this is an opportunity for affordable new experiments and projects for our students to practice optics and photonics on real world applications.

\section{ACKNOWLEDGEMENTS}

Dissemination of this work through the 2019 Education and Training in Optics (ETOP) Conference was made possible by travel funds provided by the Department of Physics and by the Office of the Provost at Kettering University.

\section{REFERENCES}

[1] Kettering University GM Mobility Center https://www.youtube.com/watch?v=Hs xsxHXM4Y

[2] SAE International, AutoDrive Challenge, https://www.sae.org/attend/student-events/autodrive-challenge/

[3] John McCarthy, "Computer Controlled Cars" - http://www-formal.stanford.edu/jmc/progress/cars/cars.html.

[4] National Highway Traffic Safety Administration (NHTSA) Letter to Google https://isearch.nhtsa.gov/files/Google\%20-\%20compiled\%20response\%20to\%2012\%20Nov\%20\%2015\%20interp\%20request\%20-$\% 204 \% 20 \mathrm{Feb} \% 2016 \% 20$ final.htm (1 May 2019)

[5] BIS Research, Emerging Technology Market Intelligence, "Global Automotive Lidar Market - Analysis and Forecast, 2018-2028", https://bisresearch.com/industry-report/automotive-lidar-market.html (1 May 2019)

[6] Research and Markets, "LIDAR technologies for the Automotive Industry: Technology benchmark, Challenges, Market forecasts", https://www.researchandmarkets.com/research/cvwt57/lidar?w=5 (1 May 2019)

[7] Strategy Analytics, "Accelerating the Future: The Economic Impact of the Emerging Passenger Economy" https://newsroom.intel.com/newsroom/wp-content/uploads/sites/11/2017/05/passenger-economy.pdf

[8] Michael E. Newman, National Institute of Standards and Technology, "To the Moon and Back in 2.5 Seconds", https://www.nist.gov/nist-time-capsule/any-object-any-need-call-nist/moon-and-back-25-seconds

[9] Tan, K.; Cheng, X., "Specular Reflection Effects Elimination in Terrestrial Laser Scanning Intensity Data Using Phong Model". Remote Sens. 9(8), 853 (2017)

[10] Frater R.H., Goss W.M., Wendt H.W. (2017) Chris Christiansen, "Telescope Design and Earth-Rotational Synthesis", in "Four Pillars of Radio Astronomy: Mills, Christiansen, Wild, Bracewell", Springer, ISBN 978-3-319-65599-4 (2017)

[11] J. C. Hulme, * J. K. Doylend, M. J. R. Heck, J. D. Peters, M. L. Davenport, J. T. Bovington, L. A. Coldren, and J. E. Bowers, "Fully integrated hybrid silicon two dimensional beam scanner", Optics Express Vol. 23(5), 5861-5874 (2015). https://doi.org/10.1364/OE.23.005861

[12] Lumotive, Beam Steering with liquid crystal metasurfaces, https://www.lumotive.com/ (1 May 2019) 